\title{
Transmission et expérience esthétique dans les premier et second degrés
}

Sylviane Ahr et Patrick Joole

\section{OpenEdition}

12 Journals

Édition électronique

URL : http://journals.openedition.org/recherchestravaux/657

DOI : 10.4000/recherchestravaux.657

ISSN : 1969-6434

Éditeur

UGA Éditions/Université Grenoble Alpes

\section{Édition imprimée}

Date de publication : 31 décembre 2013

Pagination : 133-145

ISBN : 978-2-84310-267-7

ISSN : 0151-1874

Référence électronique

Sylviane Ahr et Patrick Joole, «Transmission et expérience esthétique dans les premier et second degrés », Recherches \& Travaux [En ligne], 83 | 2013, mis en ligne le 01 juillet 2015, consulté le 08 septembre 2020. URL : http://journals.openedition.org/recherchestravaux/657 ; DOI : https://doi.org/ 10.4000/recherchestravaux.657 
Sylviane Ahr et Patrick Joole

ESPE Cergy-Pontoise

\section{Transmission et expérience esthétique dans les premier et second degrés}

De Baumgarten (Aesthetica, 1750) à Genette ("La relation esthétique» dans L'Euvre de l'art, 1997) en passant notamment par Kant ${ }^{2}$ (Critique de la faculté de juger, I79I), Schiller (Lettres sur l'éducation esthétique de l'homme, 1795), Jean-Paul (Cours préparatoire d'esthétique, 1804), Hegel (Leçons d'esthétique, I8I8-1829), l'école de Francfort (Adorno, Théorie esthétique, I970), Bourdieu (Les Règles de l'art, 1992), le terme "esthétique" ne recouvre jamais le même concept et a fortiori depuis que «les approches contemporaines de l'esthétique, en s'appuyant sur l'étymologie du terme, ont favorisé la surdétermination de l'art par la sensation ${ }^{3} »$ et privilégié de fait une esthétique de la réception ${ }^{4}$. À quel sens et à quelle théorie renvoie-t-on dès lors quand on parle d'" expérience esthétique» dans le cadre de l'enseignement littéraire de l'école au lycée et, qui plus est, quand on associe cette expression à un objectif de transmission? De quelle transmission s'agit-il par ailleurs? De la transmission d'une culture commune, d'un patrimoine littéraire commun, d'une appétence pour la lecture des œuvres littéraires, de savoirs sur les œuvres, sur l'histoire des œuvres...?

I. G. Gérard, L'Euvre de l'art, Paris, Seuil, 2010.

2. E. Kant, Critique de la faculté de juger, Paris, Gallimard, coll. "Folio Essais», I989.

3. G. Dessons; «Esthétique», dans P. Aron, D. Saint-Jacques, A. Viala (dir.), Le Dictionnaire du littéraire, Paris, Presses universitaires de France, 2002, p. 253-255, p. 255.

4. H.-R. Jauss, "Petite apologie de l'expérience esthétique», dans Pour une esthétique de la réception, Paris, Gallimard, coll. «Tel», I978, p. I35-I72. 


\section{Des ambiguïtés d'ordre institutionnel}

Le terme "esthétique» est présent dans les programmes du collège de $2008^{5}$ et dans ceux du lycée de $2010^{6}$ sans pour autant être clairement défini. Une analyse des occurrences permet cependant de constater que, dans son emploi substantivé comme adjectivé, il renvoie majoritairement à la conception qu'Alain Viala développe dans L'enseignement littéraire ${ }^{7}$ mais aussi dans $L a$ culture littéraire ${ }^{8}$ : l'expérience esthétique correspond à «des façons de voir et de ressentir", à "des appréciations subjectives"; les "émotions esthétiques sont en elles-mêmes des jugements, donc des prises de position ", qui, parce qu'elles sont "désintéressées", sont nécessairement partagées?. Cette définition s'appuie sur la conception kantienne de l'esthétique ${ }^{\mathrm{IO}}$ : le jugement de goût, subjectif et désintéressé, est devenu universel; il revient au lecteur d'identifier les critères qui ont universalisé ce jugement esthétique. On rappelle ainsi aux professeurs de collège que «choisir des textes dramatiques permet d'initier les élèves à des esthétiques variées, à différentes formes théâtrales». On étudie au lycée l'esthétique réaliste, l'esthétique classique..., et il est demandé aux professeurs de «construire au fil des lectures une connaissance des repères essentiels à la compréhension des mouvements esthétiques» dans lesquels les œuvres s'inscrivent, de privilégier "l'étude de mouvements qui marquent des étapes dans la revendication d'un renouveau esthétique», "d'apporter [aux élèves] des connaissances concernant les grands genres littéraires et leurs principales caractéristiques de forme, de sens et d'effets, afin de favoriser le développement d'une conscience esthétique». Il s'agit donc de transmettre aux élèves des "catégories d'appréciation " qui, pour Alain Viala, sont les « registres", afin qu'ils acquièrent un «jugement de goût commun» et constituent ainsi une «communauté de sensibilité entre les hommes» (Kant). Pour Hans Robert Jauss, l'expérience esthétique est en effet «amputée de ses fonctions sociales tant [...] que l'on n'introduit pas les catégories intermédiaires d'identification,

5. On relève quatre occurrences : une seule est associée à la lecture des textes littéraires, les trois autres se trouvent sous la rubrique «Histoire des arts». Programmes de français du collège, BO spécial du 28 août 2008 .

6. On relève douze occurrences. Programmes de français du lycée, $B O$ spécial du 30 septembre 2010.

7. P. Aron et A. Viala, L'Enseignement littéraire, Paris, Presses universitaires de France, coll. "Que Sais-je?», 2005, p. IO3-II2.

8. A. Viala, La Culture littéraire, Paris, Presses universitaires de France, coll. «Licence Lettres», 2009, p. 55-59.

9. Ibid.

IO. E. Kant, Critique de la faculté de juger, Paris, Gallimard, coll. «Folio Essais», I989. 
d'exemplarité et de consensus ouvert - ces catégories qui, dans l'expérience de l'art, ont été à la base de toute activité communicationnelle ${ }^{\mathrm{II}} »$. Mais l'étude des registres, associée à celle des genres, ne cadre plus les nouveaux programmes du lycée et on en comprend aisément la raison : elle nuisait à la réception effective des textes par les élèves du troisième millénaire qui, dans bien des cas, étaient invités à les lire pour retrouver les procédés visant à produire une réaction nécessairement commune à tous et inscrite dans un temps donné, celui de la première réception de l'œuvre.

La lecture des programmes du lycée invite également à s'interroger sur ce qu'il faut entendre par "développement d'une conscience esthétique». Jauss distingue trois niveaux de conscience dans l'expérience esthétique. La conscience "en tant qu'activité productrice crée un monde qui est son œuvre propre» : c'est "l'activité fictionnalisante ${ }^{\mathrm{I2}}$ " qui conduit le lecteur à produire son propre texte. La conscience «en tant qu'activité réceptrice saisit la possibilité de renouveler sa perception du monde». Enfin, l' "expérience subjective débouche sur l'expérience intersubjective - la réflexion esthétique adhère à un jugement requis par l'œuvre, ou s'identifie à des normes d'action qu'elle ébauche et dont il appartient à ses destinataires de poursuivre la définition ${ }^{13}$ ”. "Jugement requis par l'œuvre» certes, mais il revient au lecteur de réagir face à ces «normes d'action». Or ce n'est guère l'orientation prise par les rédacteurs des programmes du collège qui privilégie une conception essentialiste de la littérature. Si émotion il y a, celle-ci est inhérente au texte et non conditionnée par la réception que le lecteur en fera :

Prenant en compte la dimension esthétique d'une œuvre littéraire, l'élève développe son goût pour la musicalité et la puissance émotionnelle de la langue et, par la lecture d'œuvres littéraires, il enrichit sa connaissance de l'histoire des arts ${ }^{\mathrm{I4}}$.

C'est dans les rubriques "Histoire des arts", «Lecture de l'image» et «Lexique ${ }^{15}$ » des programmes du collège que l'on rencontre le terme «émotion» et l'expression «jugement personnel» : ils sont absents quand il est question de lecture et de littérature. Et pourtant, à la session 2013 du diplôme national du brevet, il sera demandé aux élèves de "réagir» à la lecture du texte lu en justifiant leur point de vue, compétence à évaluer également au palier 3 du socle

II. H.-R. Jauss, Pour une esthétique de la réception, ouvr. cité, p. I35-I72.

I2. G. Langlade, "Lactivité "fictionnalisante" du lecteur", dans B. Louichon et B. Laville (dir.), Les Enseignements de la fiction, Bordeaux, Presses universitaires de Bordeaux, 2006, p. I63-I77.

I3. Ibid., p. I42.

I4. Programmes de français du collège, $B O$ spécial du 28 août 2008, p. 5.

I5. Est à étudier en classe de sixième le "vocabulaire des émotions». 
commun de connaissances et de compétences : tout élève en fin de scolarité obligatoire doit être capable "après lecture de toute œuvre, [d']exprimer son point de vue de lecteur et [de] le justifier par oral ou par écrit» (compétence I). En effet, le texte de cadrage du socle précise ${ }^{16}$ :

La culture humaniste contribue à la formation du jugement, du goût et de la sensibilité.

Elle enrichit la perception du réel, ouvre l'esprit à la diversité des situations humaines, invite à la réflexion sur ses propres opinions et sentiments et suscite des émotions esthétiques.

$\mathrm{Si}$, comme le soulignent les rapporteurs de la concertation nationale «Refondons l'école ${ }^{\mathrm{I}}$ », il apparaît «essentiel de faire évoluer les programmes pour qu'ils soient en cohérence avec le socle», il semble tout autant nécessaire d'harmoniser les prescriptions institutionnelles cadrant l'enseignement littéraire dans l'ensemble des classes du secondaire. Les programmes du lycée précisent que le professeur «n'oubliera pas que la découverte du sens passe non seulement par l'analyse méthodique des différents aspects du récit qui peuvent être mis en évidence (procédés narratifs et descriptifs notamment), mais aussi par une relation personnelle au texte dans laquelle l'émotion, le plaisir ou l'admiration éprouvés par le lecteur jouent un rôle essentiel ${ }^{18}{ }^{1}$. Une relation esthétique aux œuvres littéraires ne serait-elle autorisée qu'aux lecteurs plus avertis, à ceux auxquels on a transmis les connaissances nécessaires au «développement d'une conscience esthétique permettant d'apprécier les œuvres, d'analyser l'émotion qu'elles procurent et d'en rendre compte à l'écrit comme à l'oral ${ }^{19}$ »?

Dans le texte de cadrage du socle, qui concerne les différents paliers de la scolarité obligatoire, la formation du «jugement», du "goût» et de la «sensibilité» est donc placée sur le même plan que le développement de la capacité de l'élève à réfléchir à ses propres opinions et sentiments ainsi qu'à éprouver des «émotions esthétiques». L'objectif consiste à doter les élèves du premier degré de capacités réflexives, notamment dans le cadre des activités de lecture. Dès le cycle 2, "lire ou écouter lire des œuvres intégrales, notamment de

16. Socle commun de connaissances et de compétences, Paris, CNDP / Hachette Éducation, 2008, p. 17.

17. Rapport de la concertation nationale "Refondons l'école» [en ligne], oct. 2012. Disponible sur <http://www.education.gouv.fr/cid65727/remise-du-rapport-de-la-concertation-au-president-de-la-republique.html\#Rapport_de la concertation> [consulté le Io/ıo/20I2], p. 36.

I8. C'est la seule occurrence du mot «lecteur» dans ces programmes de 20IO. De même dans ceux de 2008 pour le collège, on ne relève qu'un emploi de ce terme : le professeur développe les compétences des collégiens en lecture et "les amène progressivement à être des lecteurs autonomes", qui lisent par plaisir.

I9. Il s'agit de l'une des six finalités de l'enseignement du français et de la littérature au lycée. 
littérature de jeunesse", suppose l'existence d'un dispositif permettant à l'élève "de rendre compte de sa lecture», expression reprise et exemplifiée dans le chapitre "littérature» des programmes pour le cycle $3:$ "Les élèves rendent compte de leur lecture, expriment leurs réactions ou leurs points de vue et échangent entre eux sur ces sujets."

Cette volonté d'articuler jugement esthétique et jugement critique est l'héritage d'une tradition bien française de la lecture : souvenons-nous, par exemple, de Flaubert jugeant la fin de Graziella de Lamartine ratée car trop chargée d'émotions mensongères et proposant une réécriture plus à son goût, en prenant Voltaire comme modèle ${ }^{20}$. Pourtant les programmes restent très généraux quant à la nature exacte de cette lecture, se contentant de termes polysémiques tels que "réaction» ou "point de vue». En outre, l'absence d'explicitation du lien entre ces deux types de jugement justifie sans doute le fait que les programmes n'aient pas repris l'expression "émotion esthétique» utilisée dans le socle et qu'ils se soient contentés de souligner la nécessité de conseiller l'instauration de moments d'échange et de partage entre les élèves lecteurs. C'est reconnaître implicitement la difficulté voire l'impossibilité d'établir ce lien en littérature : "goût», «jugement " et "émotion" sont juxtaposés mais non connectés; l'injonction des programmes consistant à solliciter les « réactions des élèves» est trop générale pour supposer le projet d'une articulation entre lecture critique et lecture esthétique. Le terme "esthétique» n’est appliqué qu'aux œuvres d'art en général, en vue de définir soit leur spécificitée ${ }^{21}$ soit leur appartenance à une catégorie ou à un courant ${ }^{22}$.

Ce qui apparaît aux yeux des prescripteurs comme une impossibilité n'est pas propre aux programmes scolaires puisque le lecteur de la notice "esthétique», publiée en ligne dans l'encyclopédie Wikipedia, est également confronté à un art de l'énumération et de la juxtaposition, qui s’apparente à une stratégie embarrassée de contournement ou d'évitement :

Elle se rapporte, par exemple, aux émotions provoquées par une œuvre d'art, aux jugements de l'œuvre, à ce qui est spécifique ou singulier à une expression (artistique, littéraire, poétique, etc.), à ce qui pourrait se définir comme beau par opposition à l'utile et au fonctionnel.

20. G. Flaubert, "À Louise Colet», lettre du 24 avril I852, Correspondance, t. II (I85I-I858), Paris, Gallimard, coll. «Bibliothèque de la Pléiade», I980, p. 78.

2I. "L'enseignement des arts visuels [...] amène progressivement l'enfant à cerner la notion d'œuvre d'art et à distinguer la valeur d'usage de la valeur esthétique des objets étudiés.» (Programmes de l'école, $B O$ hors-série $\mathrm{n}^{\circ} 3$ du ig juin 2008).

22. «La sensibilité artistique et les capacités d'expression des élèves sont développées par les pratiques artistiques, mais également par la rencontre et l'étude d'œuvres diversifiées relevant des différentes composantes esthétiques, temporelles et géographiques de l'histoire des arts.» (Ibid.) 
On notera l'étrange parenté entre cette définition et ce que disent les programmes à propos de l'œuvre d'art.

De toute évidence, il serait bon, d'une part, de préciser ce à quoi les termes ou expressions "réaction", "appréciation", "point de vue personnel ", «jugement personnel », "goût», "émotion», "sentiment»..., renvoient et, d'autre part, pour l'ensemble des niveaux de la scolarité, d'harmoniser terminologie, concepts et objectifs d'enseignement et d'apprentissage.

\section{Pour lever le flou conceptuel}

Brian Stock montre comment Horace puis saint Augustin ont approfondi le lien entre la phantasia, activité imaginative qui a la forme d'une conversation intérieure, et l'émotion, lien qui fonde la dimension non seulement éthique mais aussi esthétique de la lecture ${ }^{23}$. Ce lien permet de définir un processus d'ordre phénoménologique ${ }^{24}$ qui transforme l'activité de lecture en "expérience ${ }^{25}$ » relevant de l'implication référentielle et du dialogue fantasmatique qui, pour Pierre Bayard, «en tant que position relativiste, va au rebours du courant dominant de l'esthétique que l'on pourrait qualifier de kantien ${ }^{26} »$. C'est la lecture de Mr Ramsay, décrite par son épouse dans La Promenade au phare de Virginia Woolf, qui a du mal à retenir son émotion, se tape les cuisses, tente de refouler ses larmes et finit par «rassembler tous les éléments de son appréciation », moment de "pur délice», précise-t-elle ${ }^{27}$. Bernard Stock parle alors de "relecture esthétique», Mrs Ramsay incarnant pour lui la lecture ascétique, expérience réflexive intérieure, repliée sur elle-même dans la solitude et le silence. Les contributeurs de L'Expérience de lecture en 2005, notamment Raymond $\mathrm{Michel}^{28}$, soulignent l'importance des émotions dans le cadre plus vaste de la communication "empathique et affective» avec l'auteur, au service de la rencontre de deux subjectivités.

23. B. Stock, Lire, une ascèse?, Grenoble, Jérôme Millon, 2008, p. I40.

24. M. Dufrenne, Phénoménologie de l'expérience esthétique, Paris, Presses universitaires de France, 1953, p. 432 et 487.

25. A. Trouvé, Le Roman de la lecture, critique de la raison littéraire, Bruxelles, Mardaga, 2004 .

26. P. Bayard, Comment améliorer les æuvres ratées?, Paris, Minuit, 2000, p. II9.

27. V. Woolf, La Promenade au phare, trad. M. Lanoire, Paris, Hachette, Le Livre de Poche, I983, p. I58-I59.

28. R. Michel, «Expérience de lecture et expérience esthétique : du plaisir et de l'émotion », dans V. Jouve (coord.), L'Expérience de lecture (actes du colloque de Reims), 2002, Paris, L'Improviste, 2005. 
Faisant fonctionner son "imagination et sa capacité associative au maximum ", le lecteur peut alors, nous dit Hermann Hesse, "se promener au fond de $\operatorname{soi}^{29}{ }^{2}$. Mais celui-ci prend soin de préciser qu'il s'agit là d'une étape première et provisoire : "Ce n'est plus du tout un lecteur, celui qui resterait continuellement à cette étape ne lirait plus rien.» Pour Catherine Dupuy, cette perception initiale d'un texte, qui repose sur des actions inférentielles échappant à la reconnaissance consciente, est pourtant à la fois nécessaire et incontournable ${ }^{30}$. Elle est rejointe en cela par Jean-Claude Chabanne pour qui «les lectures savantes, les lectures scolaires ne peuvent advenir que s'il y a eu lecture. Chaque lecteur commence donc là où il est. Avant la lecture il y a la lecture ${ }^{3}$. Mener les élèves sur le chemin de la lecture nécessite donc de prendre initialement appui sur une relation esthétique historiquement fondée associant imagination et émotion.

Cette relation est définie par Jean-Marie Schaeffer ${ }^{32}$ dans son essai Adieu à l'esthétique. Il précise qu'une "conduite esthétique» se caractérise par une attitude du sujet face à un objet - qui peut ne pas être artistique ou reconnu comme tel, attitude où la perception du sujet, de quelque nature qu'elle soit, est exacerbée. Cette attitude est source de plaisir - le plaisir n'étant pas incompatible avec un sentiment négatif, telle la tristesse - ou de déplaisir et elle est liée à des émotions personnelles et, dans certains cas, à une expérience personnelle. Cette attitude face à un objet est dans tous les cas une activité mentale dans la mesure où le sujet réalise des opérations de discrimination, de discernement à partir de son propre répertoire culturel et affectif. Plus ce répertoire est riche, plus les expériences esthétiques sont nombreuses.

Si l'on définit de la sorte la "conscience esthétique» que la fréquentation des œuvres littéraires (mais aussi artistiques) doit permettre aux élèves de développer, la transmission scolaire peut ne pas être réduite à celle de catégories esthétiques rendant compte d'un jugement de goût historiquement et culturellement daté, mais elle peut porter également (et peut-être davantage!) sur l'enrichissement du répertoire culturel du sujet lecteur, afin de favoriser une attitude esthétique face aux objets littéraires, ainsi que sur les outils permettant la mise en mots de l'expérience esthétique.

29. H. Hesse, «De la lecture des livres», dans Magie du livre, Paris, Corti, 1994.

30. C. Dupuy, «L'élève interprétant de littérature : quelques enjeux anthropologiques, sémiotiques et pédagogiques» [en ligne], Tréma, $\mathrm{n}^{\circ}$ 24, 2005. Disponible sur <http://trema.revues. org/688> [consulté le I4/05/20I2].

31. J.-C. Chabanne, «La lecture avant la lecture», Le français aujourd'hui, nº I2I, I998, p. 28-36, p. 28.

32. J.-M. Schaeffer, Adieu à l'esthétique, Paris, Presses universitaires de France, 2000. 


\section{Pour concilier transmission et expérience esthétique}

Le groupe départemental «Maîtrise des langages» réunissant inspecteurs, conseillers pédagogiques et IMF du premier degré, dont nous avons accompagné les réflexions, a tenté de définir des situations de lecture d'œuvres de littérature de jeunesse pour les élèves de cycle 3. Le système des personnages, la cohérence narrative et la notion d'auteur ont ainsi fait l'objet de séances prenant appui soit sur l'élaboration d'un schéma actanciel ou séquentiel soit sur une lecture en réseau intratextuel. En dépit de l'accent mis sur les outils destinés à favoriser la réussite des élèves, nous nous sommes rendu compte, lors de la réalisation effective des séances en classe, que l'enseignant ne faisait qu'imposer des savoirs relatifs aux trois domaines choisis ou transmettre sa propre lecture de l'œuvre, y compris lors des débats interprétatifs au cours desquels les propositions des élèves étaient validées à l'aune des attentes conscientes ou non de l'enseignant : l'idée que la relation esthétique s'accommodait difficilement d'une cohabitation avec un sens commun s'est alors imposée. À la priorité accordée bien involontairement à l'exhibition de ce dernier s'ajoutait le souci, bien légitime pourtant, de développer des habilités de compréhension permettant aux élèves de surmonter les obstacles supposés d'un texte. Non seulement la relation entre la spécificité littéraire de l'œuvre et l'expérience sensible du lecteur n'était pas prise en compte mais de sérieux doutes planaient sur l'amélioration des capacités des élèves à tisser une relation intime avec le texte, à simplement y entrer comme le ferait le lecteur promeneur évoqué par Julien Gracq ${ }^{33}$. Nous nous sommes alors demandé ce que pouvaient bien avoir à dire "des lecteurs démunis de savoir savant, de métalangage et de méthodes? Ont-ils seulement quelque chose à dire? Faisons le pari de les écouter. On s'apercevrait qu'ils ont déjà commencé ${ }^{4}$.»

Nous avons alors émis l'hypothèse que donner la parole aux élèves et modifier ainsi la posture de l'enseignant nécessitent de renoncer au relevé des indéterminations d'un texte jugé réticent et à l'hypervalorisation du sens qui transforme le commentaire en discours crypté et renforce le décalage entre les attentes de l'enfance et celles d'un public adulte et lettré. Dominique Bucheton ajoute, après avoir observé et décrit chacune des postures adoptées par les élèves à qui il avait été demandé de commenter un texte sans consignes particulières, que «dans les classes favorisées les enfants n'adoptent pas une posture mais

33. J. Gracq, En lisant en écrivant, Paris, José Corti, I980, dans Euvres complètes, t. II, Paris, Gallimard, coll. «Bibliothèque de la Pléiade», I995.

34. J.-C. Chabanne, «La lecture avant la lecture», ouvr. cité. 
plusieurs» et que "dans les classes plus défavorisées, les enfants ont tendance à se limiter à deux postures ${ }^{35}$ ». C'est cette posture démultipliée qui permet aux élèves de penser par eux-mêmes au lieu de répéter les mots des autres.

La double nécessité de prendre en compte une expérience d'ordre esthétique et de démultiplier la posture de l'élève a conduit à l'élaboration d'une séquence en trois temps. Après lecture de l'œuvre ${ }^{36}$, les élèves s'expriment sur leur ressenti, d'abord par écrit et individuellement, puis échangent ensemble sur leurs impressions respectives et les causes possibles de leur ressenti après que leurs écrits ont été affichés dans la classe. Dans un second temps, une phase de catégorisation gérée par l'enseignant permet aux élèves de regrouper leurs propositions et ainsi de faire apparaître des éléments de caractérisation de l'œuvre. L'enseignant fait ensuite lire d'autres œuvres permettant aux élèves de se demander s'ils ressentent ou non les mêmes impressions et les mêmes émotions. La comparaison avec d'autres livres d'un même auteur est particulièrement intéressante car elle permet de se demander s'il existe une possible cohérence liée à des effets plus ou moins intentionnels.

Non seulement la libre parole des élèves est accueillie et la relation esthétique encouragée mais la posture professionnelle de l'enseignant s'en trouve modifiée. Il s'agit moins pour lui de préparer la lecture des élèves en faisant des recherches sur l'œuvre et son auteur que d'essayer d'en cerner la spécificité au fur et à mesure des propositions des élèves. Il est alors disponible pour les écouter et prendre en compte leur réception effective. Mais libre à lui, après la phase de catégorisation, de se rassurer en consultant des commentaires autorisés mais, comme le dit l'une des conseillères pédagogiques faisant partie du groupe départemental, "ce savoir n’est pas transmis ni imposé aux élèves, il permet seulement à l'enseignant de les accompagner».

Nous avons nous-mêmes observé, lors de l'expérimentation de notre séquence dans des écoles du Val d'Oise situées en particulier dans des zones d'éducation prioritaire, une implication enjouée de tous les élèves, y compris de ceux en difficulté ou habituellement en retrait. L'expérience esthétique, liée à la sollicitation imaginaire et émotionnelle, était bien ce moment de "pur délice» (Woolf) ou de «jouissance» (Jauss) que des chercheurs d'origines

35. D. Bucheton, Devenir l'auteur de sa parole [en ligne], ministère de l'Éducation nationale - Direction de l'enseignement scolaire, 2002. Disponible sur <http://www.crdp.accreteil.fr/ langages/rubriques/pdf/contributions_reflexion/parole_bucheton_Io9870.pdf> [consulté le $25 / 05 / 2012]$.

36. Effectuée oralement par l'enseignant ou individuellement par l'élève soit avec le livre soit par l'intermédiaire du vidéoprojecteur. 
aussi diverses que Béatrice Bloch ${ }^{37}$, Marilyn Brault ${ }^{38}$, Patricia Limido-Heulot ${ }^{39}$ décrivent en termes presque semblables.

Mais par-delà le réel plaisir de lire favorisé par «ce discours organisé de la communication intime avec un livre» souhaité par Julien Gracq ${ }^{40}$ nous nous sommes demandé s'il était possible, grâce à l'expérience esthétique, de favoriser l'émergence d'habilités favorisant la mise en mémoire des œuvres lues et par conséquent la constitution d'une bibliothèque intérieure. Patricia LimidoHeulot et Mikel Dufrenne émettent des doutes quant à l'existence de souvenirs persistants de lecture liés à une expérience esthétique, qu'ils définissent à la manière d'Ingarden comme "une totalité de vie séparée ${ }^{41}$ ». Brigitte Louichon rappelle que le lecteur se souvient de l'objet livre, de l'effet, des circonstances de la lecture et finalement très peu du texte ${ }^{42}$. C'est la mémoire "oublieuse" dont parlent Francis Marcoin en $2004^{43}$ et le romancier Murakami en $2008^{44}$ : en dépit du fait que l'héroïne de la nouvelle intitulée «Le sommeil» ne retrouve pas de souvenirs précis, elle sait que néanmoins quelque chose de cet ancien jeu intentionnel a subsisté et s'est déposé en elle. Alors faute de retrouver en sa mémoire consciente de telles traces, son corps se souvient pour elle et l'invite à la gourmandise, la presse de manger du chocolat car autrefois sa lecture (dont elle a oublié le contenu singulier) en était ainsi accompagnée et rythmée.

L'enrichissement du répertoire culturel passe donc par la mise en mémoire non seulement des objets mais aussi et surtout des expériences esthétiques qui leur sont liées. Tout lecteur, novice ou expert, se souvient des livres qui l'ont particulièrement marqué, positivement ou négativement, et cela, souvent

37. B. Bloch, «Imaginaire dans l'expérience esthétique de la lecture», dans Esthétique plurielle, Saint-Denis, Presses universitaires de Vincennes, 1996.

38. M. Brault, "Le rôle de l'imaginaire dans l'expérience esthétique de la lecture littéraire au collégial", dans M. Roy, M. Brault et S. Brehm (éd.), Formation des lecteurs : formation de l'imaginaire [en ligne], Figura, Centre de recherche sur le texte et l'imaginaire, Montréal, université du Québec à Montréal, 2008. Disponible sur <http://oic.uqam.ca/ sites/oic.uqam. ca/files/oici/cf2O-5-brault-role_imaginaire.pdf> [consulté le Io/05/20I2].

39. P. Limido-Heulot, «L’expérience esthétique, entre feinte intentionnelle et épreuve réelle», dans Bulletin d'analyse phénoménologique [en ligne], vol. VI, ${ }^{\circ}$ 7, 2010. Disponible sur $<$ http:// popups.ulg.ac.be/bap/docannexe.php?id=42I> [consulté le 2/o6/20I2].

40. J. Gracq, En lisant en écrivant, ouvr. cité, p. 676.

4I. R. Ingarden, La Connaissance de l'œuvre d'art littéraire, Tübingen, Niemeyer, I968, p. 23I.

42. B. Louichon, "La mémoire lettrée», dans D. Dubois-Marcoin et C. Tauveron (coord.), Français, langue et littérature, socle commun. Une culture pour les élèves? Quelle professionnalité pour les enseignants? (actes du colloque, Lyon, I2-I4 mars 2008) [en ligne]. Disponible sur <http://ife.ens-lyon.fr/editions/editions-electroniques/francais-langue-et-litterature-soclecommun> [consulté le I8/05/20I2].

43. F. Marcoin, «La question du littéraire», dans Nouveaux Regards sur la lecture, Observatoire national de la lecture, Scérén-CNDP, 2005.

44. H. Murakami, L'Éléphant sévapore, Paris, Belfond, 2008. 
de façon parcellaire voire métonymique, comme Pierre Bayard l'explique en prenant pour exemple le défaut de mémoire de Montaigne ${ }^{45}$. Dans l'article "Génie" de l'Encyclopédie, Saint-Lambert précise que "lorsque l'âme a été affectée par l'objet même, elle l'est encore par le souvenir ${ }^{46}{ }^{4}$. Entendons par "âme» la sensibilité du lecteur et par «répertoire culturel», la «bibliothèque intérieure", cette "partie subjective de la bibliothèque collective comportant les livres marquants de chaque sujet ${ }^{47}$ ». Dans un tel cas, la transmission ne vise pas seulement l'acquisition, à l'école, d'une première culture littéraire, au collège, d'une "culture nécessaire à la compréhension des œuvres littéraires» et, au lycée, «la connaissance des grands genres littéraires, de leurs principales caractéristiques de forme, de sens et d'effets». Mais la transmission vise également la construction de cette bibliothèque intérieure, personnelle, avec laquelle chaque sujet lecteur met en résonance les lectures nouvelles qu'il réalise et qui l'aide à écrire ce "texte que nous écrivons en nous quand nous lisons».

C'est pourquoi, "apprendre à dire-écrire l'expérience esthétique et pas seulement à la commenter ${ }^{4}$ ", créer "l'événement» de la lecture dont on sait à quel point il est important dans la constitution d'une mémoire et solliciter cette "dimension affective de la mémoire personnelle ${ }^{49}$ » exigent aussi la tenue d'un carnet de lecteur s'introduisant "dans la place de l'auteur", insinuant "les ruses du plaisir et une réappropriation dans le texte de l'autre», geste indispensable pour Michel de Certeau à "l'invention de mémoire ${ }^{50}$ ». Il nous paraît donc important d'encourager les processus de prélèvements et d'insertions en développant, dans le carnet, les gestes d'annotations, de recopiages et de reformulations en lieu et place de résumés.

Les expérimentations menées dans le secondaire autour de l'utilisation du cahier de lecture littéraire en lien avec la pratique du débat favorisent, comme à l'école élémentaire, la construction et la conservation du répertoire culturel et affectif personnel, indispensable à l'expérience esthétique.

45. P. Bayard, Comment parler des livres que l'on n'a pas lus?, Paris, Minuit, 2007, p. 55-62. 46. 1757, p. 582.

47. P. Bayard, ouvr. cité, note 45, p. 74, note I6.

48. J.-C. Chabanne, "Â la recherche du lecteur empirique, problématiques», conférence d'ouverture des $\mathrm{V}^{\text {es }}$ Rencontres de didactique de la littérature, Parler, lire, écrire dans la classe de littérature : l'activité de l'élève / le travail de l'enseignant / la place de l'œuvre [en ligne], IUFM de Montpellier, 6 au 8 avril 2006. Disponible sur <http://chabanne.jeancharles.perso.neuf.fr/ publis/7eRdidlit_Mpt_conf.pdf>[consulté le I4/05/20I2].

49. V. Jouve, «La lecture comme retour sur soi : de l'intérêt pédagogique des lectures subjectives", dans A. Rouxel et G. Langlade (dir.), le sujet lecteur - Lecture subjective et enseignement de la littérature, Rennes, Presses universitaires de Rennes, 2004, p. IO5-II4, p. Io6.

50. M. de Certeau, L'Invention du quotidien, t. I, Paris, Gallimard, coll. «Arts de faire», I990. 
Le cahier de lecture littéraire ${ }^{\text {sI }}$ donne, dans un premier temps, la priorité à la réception effective du texte par les sujets lecteurs. La mise en mots de cette réception est facilitée et favorisée par un questionnement professoral tout autre que celui ordinairement proposé dans les classes. Les questions et consignes sollicitent et/ou favorisent l'engagement des élèves dans la lecture du texte, leur appréciation (axiologique, esthétique), la mise en mots des sensations (auditives, visuelles...) éprouvées à la lecture, l'émergence des images mentales que le lecteur se fabrique au moment de sa lecture, le processus d'identification qui peut s'opérer parfois, ainsi que le vécu, l'expérience, la «bibliothèque intérieure» de chaque lecteur. Le retour au cahier après les échanges intersubjectifs invite les élèves à interroger leur "attitude esthétique" première au regard de celle de leurs pairs. Dans le sillage de Gérard Genette, on considère en effet que «le jugement esthétique est "sans appel", c'est-à-dire autonome et souverain» et que «seule une évolution intérieure" est susceptible de le modifier ${ }^{52}$. Le cahier garde trace de ces évolutions intérieures, signes d'une «formation du jugement et de l'esprit critique» en cours, formation qui constitue l'une des six finalités de l'enseignement du français au lycée.

En d'autres termes, le cahier est non une anthologie des objets lus mais la mémoire des lectures personnelles : il rend compte des expériences esthétiques des sujets lecteurs au répertoire affectif, expérientiel, cognitif, linguistique et culturel singulier. Les interactions au moment du débat favorisent non seulement la mise en mots de ces expériences esthétiques mais également l'enrichissement de ce répertoire personnel, ce dont l'analyse des cahiers rend compte. Il s'agit dès lors de transmettre non "des modèles sémiotiques ou narratologiques ${ }^{53}$ ” mais les outils linguistiques et intellectuels permettant aux sujets lecteurs d'appréhender le sensible et, par là même, de leur assurer le développement d'une conscience esthétique.

5I. Objet ainsi désigné pour souligner sa nature scolaire et répondre à la conception de la lecture littéraire qui sous-tend la démarche, à savoir un processus dialectique qui requiert de la part du sujet lecteur une compétence empathique et une compétence analytique. S. Ahr et P. Joole (dir.), «Carnet et journal de lecture : état de la question» (actes de la Journée d'étude internationale du 23mai 20I2) IUFM/université de Cergy-Pontoise, Namur, Presses universitaires de Namur, coll. "Diptyque», 2013; S. Ahr, "Le carnet de lecteur de littérature au collège et au lycée : un dispositif expérimental misant sur une nouvelle approche de l'interaction lectureécriture», dans S. Aeby (dir.), coll. «Diptyque», 2I, Namur, Presses universitaires de Namur, 20II, p. I57-I78; P. Joole "Le carnet de lecture : un outil d'enseignement et de formation", dans I. de Peretti et B. Ferrier (dir.), Enseigner les classiques aujourd'hui, Bruxelles, Peter Lang, 20I2, p. 259-273.

52. G. Genette, L'Euvre de l'art, Paris, Seuil, 20I0, p. 495.

53. M. Macé, Façons de lire, manières d'être, Paris, Gallimard, coll. «NRF Essais», 20II, p. I5. 
TRANSMISSION ET EXPÉRIENCE ESTHÉTIQUE DANS LES PREMIER ET SECOND DEGRÉS

L'acte de lecture se nourrit des sentiments et des émotions du lecteur, comme le souligne Alberto Manguel : "Nous lisons avec mépris, avec admiration, avec négligence, avec colère, avec passion, avec envie, avec nostalgie ${ }^{54}$." Les programmes, bien qu'ambigus, laissent néanmoins la possibilité de mettre en place des dispositifs qui prennent l'expérience esthétique comme point de départ afin que la transmission des œuvres puisse s'effectuer délicieusement et durablement.

54. A. Manguel, Une histoire de la lecture, Arles, Actes Sud, I998, p. 357. 